\title{
Networks of Interacting Machines \\ Production Organization in Complex Industrial Systems and Biological Cells
}




\title{
WORLD SCIENTIFIC LECTURE NOTES IN COMPLEX SYSTEMS
}

Editor-in-Chief: A.S. Mikhailov, Fritz Haber Institute, Berlin, Germany

H. Cerdeira, ICTP, Triest, Italy

B. Huberman, Hewlett-Packard, Palo Alto, USA

K. Kaneko, University of Tokyo, Japan

$\mathrm{Ph}$. Maini, Oxford University, UK

\begin{abstract}
AIMS AND SCOPE
The aim of this new interdisciplinary series is to promote the exchange of information between scientists working in different fields, who are involved in the study of complex systems, and to foster education and training of young scientists entering this rapidly developing research area.

The scope of the series is broad and will include: Statistical physics of large nonequilibrium systems; problems of nonlinear pattern formation in chemistry; complex organization of intracellular processes and biochemical networks of a living cell; various aspects of cell-to-cell communication; behaviour of bacterial colonies; neural networks; functioning and organization of animal populations and large ecological systems; modeling complex social phenomena; applications of statistical mechanics to studies of economics and financial markets; multi-agent robotics and collective intelligence; the emergence and evolution of large-scale communication networks; general mathematical studies of complex cooperative behaviour in large systems.
\end{abstract}

\section{Published}

Vol. 1 Nonlinear Dynamics: From Lasers to Buttertlies

Vol. 2 Emergence of Dynamical Order: Synchronization Phenomena in Complex Systems 
World Scientific Lecture Notes

in Complex Systems - Vol. 3

editors

Dieter Armbruster

Arizona State University, USA

Kunihiko Kaneko

University of Tokyo, Japan

Alexander S. Mikhailov

Fritz Haber Institute, Germany

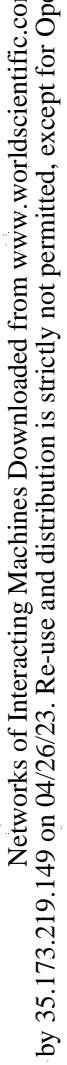

Networks of

Interacting Machines

Production Organization in Complex Industrial Systems and Biological Cells 


\section{Published by}

World Scientific Publishing Co. Pte. Ltd.

5 Toh Tuck Link, Singapore 596224

USA office: 27 Warren Street, Suite 401-402, Hackensack, NJ 07601

UK office: 57 Shelton Street, Covent Garden, London WC2H 9HE

\section{British Library Cataloguing-in-Publication Data}

A catalogue record for this book is available from the British Library.

\section{NETWORKS OF INTERACTING MACHINES \\ Production Organization in Complex Industrial Systems and Biological Cells}

Copyright $\odot 2005$ by World Scientific Publishing Co. Pte. Ltd.

All rights reserved. This book, or parts thereof, may not be reproduced in any form or by any means, electronic or mechanical, including photocopying, recording or any information storage and retrieval system now known or to be invented, without written permission from the Publisher.

For photocopying of material in this volume, please pay a copying fee through the Copyright Clearance Center, Inc., 222 Rosewood Drive, Danvers, MA 01923, USA. In this case permission to photocopy is not required from the publisher.

ISBN $981-256-498-5$ 


\section{PREFACE}

This book is devoted to a discussion of analogies and differences of complex production systems - natural, as in biological cells, or man-made, as in economic systems or industrial production. Hierarchical models of industrial supply chains are characterized by systems of differential equations closely related to traffic flow models. Interesting questions asked in this context go much beyond the static network analysis. Since most production systems are highly stochastic and event-driven, they are rarely, if ever, at equilibrium. Hence the reaction of a given supply network to specific events as well as its transient response to parameter changes and control actions has to be understood through simulation as well as through fundamental models. Additionally, these dynamic responses are constrained by economics, leading to the evolution of systems with higher productivity and profitability. On the other hand, recent research has put forward a view of a biological cell as a factory where products of one machine are used by other machines for manufacturing of their own products or for regulation of their functions. In some cases, a cell may operate in a synchronous mode, so that the operation cycles of individual machines are temporally correlated and the intermediate parts are released exactly when they are needed for further production by other machines. A special property of biological production networks is that, to a large extent, their activity is self-organized and persists despite the presence of strong thermal fluctuations. Progress in micro- and nanotechnology may soon bring about a possibility to produce nanorobots and to design selfreproducing artificial cells, leading to a revolution in industrial manufacturing. However, the principles of purposeful operation of large ensembles of nanodevices and populations of artificial cells should first be investigated. Obviously, they again should bear strong similarities to biological organization. The common themes of industrial and biological production include evolution and optimization, synchronization and self-organization, robust operation despite high stochasticity, and hierarchical dynamics. 
The book presents selected lectures given at the international workshop "Networks of Interacting Machines: Industrial Production Systems and Biological Cells" (Berlin, December 2003), organized with the financial support of the Klaus Tschira Foundation. Its authors are a group of scientists and industrialists from Europe, Japan and USA. Together, we hope to provide an overview of modern perspectives on principles of production organization.We are grateful to the Tschira Foundation for providing financial support and thank Dr. Oliver Rudzick for his assistance in the preparation of manuscripts.

\author{
Dieter Armbruster \\ Arizona State University \\ Tempe, AZ, USA \\ Kunihiko Kaneko \\ University of Tokyo \\ Tokyo, Japan
}

Alexander S. Mikhailov Fritz-Haber-Institut der Max-Planck-Gesellschaft Berlin, Germany 


\section{CONTENTS}

Preface $\quad$ v

1 Continuum Models for Interacting Machines 1

Dieter Armbruster, Pierre Degond, Christian Ringhofer

1.1 Introduction $\ldots \ldots \ldots \ldots \ldots \ldots$

1.2 Heuristic Models . . . . . . . . . . . . . . . . . 5

1.2.1 Quasistatic Models . . . . . . . . . . . . . . 5

1.2.2 Advection-Diffusion Equations . . . . . . . . 8

1.2.3 Policies and Bottlenecks . . . . . . . . . . . 11

1.2.3.1 Dispatch Rules . . . . . . . . . . . . . 11

1.2.3.2 Bottlenecks and Maximal Capacities .... 12

1.3 First Principle Models . . . . . . . . . . . . . . . . . . . . . 13

1.3.1 Kinetic Models . . . . . . . . . . . . . . . . 13

1.3.2 Deterministic Kinetic Models . . . . . . . . . . . . 14

1.3.3 Stochasticity and Diffusion . . . . . . . . . . 21

1.4 Conclusions . . . . . . . . . . . . . . . . . . . . 29

References ..................... 31

2 Supply and Production Networks: From the Bullwhip Effect to Business Cycles

Dirk Helbing, Stefan Lämmer

2.1 Introduction . . . . . . . . . . . . . . 34

2.2 Input-Output Model of Supply Networks . . . . . . . . 35

2.2 .1 Adaptation of Production Speeds . . . . . . . . . 36

2.2.2 Modelling Sequential Supply Chains . . . . . . . . 37

2.2.3 More Detailed Derivation of the Production Dynamics 39

2.2.4 Dynamic Solution and Resonance Effects . . . . . 40

2.2.5 The Bullwhip Effect . . . . . . . . . . . . 41 
2.3 Network Effects . . . . . . . . . . . . . . . . 43

2.3.1 General Methods of Solution . . . . . . . . . . 44

2.3.2 Examples of Supply Networks . . . . . . . . . . 47

2.4 Network-Induced Business Cycles . . . . . . . . . . . 51

2.4.1 Treating Producers Analogous to Consumers . . . . 53

2.5 Reproduction of Some Empirically Observed Features of Business Cycles ... . . . . . . . . . . . . 54

2.5.1 Dynamic Behaviors and Stability Thresholds . . . 55

2.6 Summary . . . . . . . . . . . . . . . . 59

2.7 Future Research Directions . . . . . . . . . . . . 60

2.7 .1 Network Engineering . . . . . . . . . . . . . 60

2.7.2 Cyclic Dynamics in Biological Systems . . . . . . . 60

2.7.3 Heterogeneity in Production Networks . . . . . . . . 61

2.7.4 Multi-Goal Control . . . . . . . . . . . . . . . 61

2.7.5 Non-Linear Dynamics and Scarcity of Resources . . 61

Appendix A. . . . . . . . . . . . . . . . . 63

2.8 Boundary between Damped and Growing Oscillations . . . 63

2.9 Boundary between Damped Oscillations and Overdamped Behavior .................... 64

References .................... 64

3 Managing Supply-Demand Networks in Semiconductor Manufacturing Karl Kempf

3.1 Introduction to Supply-Demand Networks . . . . . . . . 67

3.2 Examples from the Semiconductor Manufacturing . . . . 69

3.2.1 A Product-Centric Perspective . . . . . . . . . 70

3.2 .2 A Facilities-Centric Perspective . . . . . . . . . . 71

3.2.3 Repetitive Decisions . . . . . . . . . . . . . 72

3.2 .4 Combinatorial Complexity . . . . . . . . . . . 73

3.2.5 Complexity from Supply Stochasticity . . . . . . . 73

3.2.6 Complexity from Demand Stochasticity . . . . . . 74

3.2.7 Complexity from Nonlinearity . . . . . . . . . . . . . 75

3.2.8 Financial Complexity . . . . . . . . . . . 75

3.3 Managing Supply-Demand Networks . . . . . . . . . . 76

3.3.1 A Capacity Planning Formulation . . . . . . . . 77 
3.3.2 An Inventory Planning Formulation $\ldots \ldots \ldots$

3.3.3 Integrating Capacity and Inventory Planning . . . 86

3.3.4 A Tactical Execution Formulation . . . . . . . . 88

3.3.5 Simulation Support . . . . . . . . . . . . 94

3.4 Conclusions . . . . . . . . . . . . . . . . . 97

References . . . . . . . . . . . . . . . . 98

4 Modelling Manufacturing Systems for Control:

A Validation Study

Erjen Lefeber, Roel van den Berg, J.E. Rooda

4.1 Introduction . . . . . . . . . . . . . . . . 101

4.2 Preliminaries . . . . . . . . . . . . . . . 102

4.3 Effective Process Times (EPT's) . . . . . . . . . . 105

4.4 Control Framework . . . . . . . . . . . . . . . . 108

4.4 .1 Approximation Model . . . . . . . . . . . . 109

4.4.2 Model Predictive Control (MPC) . . . . . . . . . 110

4.4.3 Control Framework (revisited) . . . . . . . . . 111

4.5 Modelling Manufacturing Systems . . . . . . . . . . 113

4.6 Validation of PDE-Models . . . . . . . . . . . 115

4.6.1 Manufacturing Systems . . . . . . . . . . 116

4.6 .2 PDE-Models . . . . . . . . . . . . . . 117

4.6 .3 Validation Study . . . . . . . . . . . . . . 118

4.7 Concluding Remarks . . . . . . . . . . . . . . . . 122

References ...................... . . 125

5 Adaptive Networks of Production Processes 127 Adam Ponzi

5.1 Introduction . . . . . . . . . . . . . . 127

5.2 Review of von-Neumann Model . . . . . . . . . . . 130

5.3 Dynamical Production Model . . . . . . . . . . . . . . . . 132

5.4 Model Behaviour . . . . . . . . . . . . . . . . 136

5.4.1 Single Process in Fixed Environment . . . . . . . 136

5.4 .2 Multiple Timescales . . . . . . . . . . . . . . 141

5.4 .3 Complex Dynamics . . . . . . . . . . . . . . . 143

5.4 .4 Network Structure . . . . . . . . . . . . . 148

5.5 Discussion . . . . . . . . . . . . . . . . 150

References ....................... 153 
6 Universal Statistics of Cells with Recursive Production 155 Kunihiko Kaneko, Chikara Furusawa

6.1 Question to be Addressed . . . . . . . . . . . . . 155

6.2 Logic . . . . . . . . . . . . . . . . . . . . 156

6.3 Model . . . . . . . . . . . . . . . . . . . . . . . . 159

6.4 Zipf Law . . . . . . . . . . . . . . . . . . 163

6.5 Log-Normal Distribution . . . . . . . . . . . . 167

6.6 Experiment . . . . . . . . . . . . . . . . 171

6.6.1 Confirmation of Zipf Law . . . . . . . . . . . 171

6.6.1.1 Confirmation of Laws on Fluctuations . . . 171

6.7 Discussion . . . . . . . . . . . . . . 172

References .................... 175

7 Intracellular Networks of Interacting Molecular Machines

Alexander S. Mikhailov

7.1 Introdution . . . . . . . . . . . . . . 177

7.2 Networks of Protein Machines . . . . . . . . . . . 180

7.3 Coherent Molecular Dynamics . . . . . . . . . . . . 184

7.4 Mean-Field Approximation . . . . . . . . . . . . . 192

7.5 Further Theoretical Developments . . . . . . . . . . . 196

7.6 Coherence in Cross-Coupled Dynamical Networks . . . . 198

7.7 Discussion . . . . . . . . . . . . . . . . . . 201

References .................... . 202

8 Cell is Noisy 203 Tatsuo Shibata

8.1 Introduction . . . . . . . . . . . . . . . . 203

8.2 Origin of Molecular Noise . . . . . . . . . . . . . 204

8.3 Stochastic Gene Expression . . . . . . . . . . . . . . 206

8.3.1 Noise in Single Gene Expression . . . . . . . . 207

8.3.2 Attenuating Gene Expression Noise by Autoregulation . . . . . . . . . . 210

8.4 Noisy Signal Amplification in Signal Transduction

Reactions . . . . . . . . . . . . . . . . . 212

8.5 Propagation of Noise in Reaction Networks . . . . . . . 215

8.6 Outlook . . . . . . . . . . . . . . . . . 218

References . . . . . . . . . . . . . . . . 218 


\section{An Intelligent Slime Mold: A Self-Organizing}

System of Cell Shape and Information

\section{Tetsuo Ueda}

9.1 Introduction . . . . . . . . . . . . . . . 221

9.1.1 The True Slime Molds, Like Nothing on Earth . . . 222

9.2 Cell Motility and Cell Behavior by the Plasmodium . . . . 223

9.2 .1 Cell Motility . . . . . . . . . . . . . . . 223

9.2 .2 Chemotaxis . . . . . . . . . . . . . . . . . 224

9.2.3 Sensing and Transduction . . . . . . . . . . 225

9.2.4 Search for Second Messengers . . . . . . . . . . 226

9.3 Integration of Sensed Information in Chemotaxis . . . . . 226

9.3.1 A Model for Integration . . . . . . . . . . . . . 228

9.4 Collective Dynamics of Coupled Oscillators in Cell Behavior 229

9.4.1 The Response to External Stimulation . . . . . . . 230

9.4.2 Alteration of the Judgment by Oscillatory Stimulation through Entrainment . . . . . . . . . 232

9.4.3 Correspondence of Tactic Behavior with Contractility 233

9.4.4 Bifurcation of Dynamic States in the Feeding Behavior by the Placozoan . . . . . . . . . . . . . 234

9.5 Chemical Oscillations as a Basis for the Rhythmic Contraction234

9.6 Transition of Chemical Patterns Accompanying the Selection of Cell Behavior . . . . . . . . . . . . . 235

9.6.1 Theory of Cell Behavior in Terms of Dissipative Structure . . . . . . . . . . . . 237

9.6.2 Link to the Organization of Cytoskeleton and Chemical Pattern . . . . . . . . . . . . . 237

9.7 Computing by Changing Cell Shape . . . . . . . . . 238

9.7.1 Solving a Maze Problem . . . . . . . . . . . . . 239

9.7.2 Solving the Steiner Problem . . . . . . . . . . . . 240

9.7.3 Formation of Veins by External Oscillation . . . . . 240

9.8 Fragmentation of the Plasmodium: Control of Cell Size . . 242

9.8.1 Thermo-Fragmentation . . . . . . . . . . . . 242

9.8.2 Photofragmentation and its Photosystem . . . . . 243

9.9 Memory Effects and Morphogen: Phytochrome as Morphogen in the Fragmentation . . . . . . . . . . . . . . 244

9.10 Locomotion . . . . . . . . . . . . . . . . . . 247

9.10 .1 Allometry in Locomotion Velocity . . . . . . . 247 
9.10.2 Correlation of Oscillations During Directional Movement . . . . . . . . . . . . . . . . 247

9.11 The Emergence of the Rhythmic Streaming . . . . . . . . . 248

9.12 Time Order Among Multiple Rhythms in the Plasmodium ... . . . . . . . . . . . . 250

9.12.1 Long-Term Changes in Cell Shape of the Plasmodium . . . . . . . . . . . . 2 250

9.12.2 Multiple Oscillations . . . . . . . . . . . . . . . 250

9.13 Concluding Remarks as Future Prospects . . . . . . . . 251

References . . . . . . . . . . . . . . . 253

10 Communication and Structure within Networks 257 Kim Sneppen, Martin Rosvall, Ala Trusina

10.1 Introduction . . . . . . . . . . . . . . . . 257

10.2 An Economy for Exchange of Social Contacts . . . . . . . 258

10.3 Limited Information Horizons in Complex Networks . . . . 261

10.4 Conclusion . . . . . . . . . . . . . . 266

References . . . . . . . . . . . . . . . 267 